\title{
Systolic time intervals in atrial fibrillation and mitral stenosis
}

\author{
Paul Kligfield ${ }^{1}$ \\ From the Cardiac Department, St. George's Hospital, London S.W.I
}

The duration of left ventricular ejection was related to the preceding $R R$ interval in $I 5$ patients with varying duration of diastole, and hence ventricular filling time, from atrial fibrillation. Eleven had varying degrees of mitral stenosis.

Left ventricular ejection time lengthened in relation to increasing $R R$ intervals, but reached a duration beyond which no further significant increase in ejection time occurred despite a further increase in the $R R$ interval.

The $R R$ interval needed to achieve maximum left ventricular ejection and the slope of the curve at short cycle lengths could be related to the presence and severity of mitral stenosis as measured by echocardiography.

The pre-ejection period decreased with increasing $R R$ interval in 9 patients in whom it was measured.

The ratio of pre-ejection to left ventricular ejection period decreased rapidly with increasing $R R$ interval to reach a minimum value beyond which further increase in $R R$ interval produced no significant change.

Most studies of systolic time interval are concerned with deriving average values for groups of subjects rather than examining relative changes in an individual patient (Weissler, Harris, and Schoenfeld, I969).

Previous measurements of left ventricular ejection time in atrial fibrillation in groups of patients have shown that an inverse relation with the preceding cycle length exists (Katz and Feil, 1923; Schoenfeld et al., 1963) and that left ventricular ejection can be correlated with stroke volume (Greenfield et al., 1968). Recently, Tavel et al. (1972) have observed that in the absence of mitral stenosis, rising left ventricular ejection in individual patients tends to level off as cycle lengths approach $850 \mathrm{msec}$, suggesting completion of ventricular filling, but no similar trend was noted for patients with mitral stenosis.

In patients with mitral stenosis and atrial fibrillation, ventricular filling should be completed with sufficiently long diastole. It is shown in this study that in these patients $R R$ intervals exist beyond which no further significant increase in left ventricular ejection time occurs. Since obstruction to

Received 4 March 1974.

1 Present address: Division of Cardiology, Department of Medicine, New York Hospital - Cornell Medical Center, 525 East 68 Street, New York, N.Y. I0021, U.S.A. left ventricular inflow in mitral stenosis might be expected to alter the pattern of ventricular filling during early diastole, features of the left ventricular ejection curves are defined which allow comparison of individual patients. Variation of pre-ejection period and the ratio of pre-ejection period to left ventricular ejection time with changing cycle length for individual patients in atrial fibrillation is also demonstrated.

\section{Methods}

The characteristics of the patients studied are presented in the Table. All patients were in atrial fibrillation, and all except one were given digitalis. Of the $I$ I patients with mitral stenosis, 8 had additional slight mitral regurgitation and 3 also had slight aortic regurgitation. Four patients had no clinical or echocardiographic evidence of mitral stenosis: I had ischaemic heart disease, I had severe chronic mitral regurgitation of non-rheumatic aetiology, and 2 had lone atrial fibrillation.

Simultaneous high frequency phonocardiograms, electrocardiograms, and external carotid pulse tracings were recorded at a paper speed of $100 \mathrm{~mm} / \mathrm{sec}$ using a Cambridge multichannel photographic recorder. All recordings were obtained with the patient in a semisupine position with an approximately $30^{\circ}$ elevation of the head and trunk.

Time intervals were measured to the nearest ro msec, according to standard technique (Weissler et al., 1969). 
TABLE Characteristics of patients studied

\begin{tabular}{|c|c|c|c|c|c|c|c|c|c|}
\hline Case no. & Sex & $\begin{array}{c}\text { Age } \\
(y r)\end{array}$ & Diagnosis & $N Y H A$ & $R R$ range & $' T '$ & ' $S$ ' & $R m v$ & $\begin{array}{l}\text { Diastolic } \\
\text { closure } \\
\text { rate }\end{array}$ \\
\hline $\mathbf{I}$ & $\mathbf{F}$ & $6 I$ & $\begin{array}{l}\text { Mitral stenosis and regurgitation, } \\
\text { aortic regurgitation }\end{array}$ & III & $800-1500$ & 1250 & 212 & $5 \cdot 9$ & 0.9 \\
\hline 2 & $\mathbf{F}$ & 65 & Mitral stenosis and regurgitation & II & $740-1380$ & 850 & 352 & $2 \cdot 4$ & $3 \cdot 0$ \\
\hline 3 & $\mathbf{F}$ & 56 & Mitral stenosis and regurgitation & II & $410-810$ & 650 & 392 & $1 \cdot \dot{6}$ & $2 \cdot 5$ \\
\hline 4 & & & aortic regurgitation & III & $430-136 n$ & 915 & 312 & $2 \cdot 9$ & $2 \cdot 0$ \\
\hline 5 & $\mathbf{F}$ & 58 & Mitral stenosis & II & $600-1380$ & 1025 & 212 & $4 \cdot 8$ & $1 \cdot 6$ \\
\hline 6 & $\mathbf{M}$ & 55 & Mitral stenosis and regurgitation & I & $490-1320$ & 800 & 228 & 3.5 & $3 \cdot 0$ \\
\hline 7 & $\mathrm{~F}$ & 44 & Mitral stenosis & II & $510-1140$ & 890 & 216 & $4 \cdot 1$ & $I \cdot 6$ \\
\hline 8 & $\mathbf{M}$ & 53 & $\begin{array}{l}\text { Mitral stenosis and regurgitation, } \\
\text { aortic regurgitation }\end{array}$ & III & $610-1580$ & ro5o & 228 & $4 \cdot 6$ & $I \cdot 5$ \\
\hline 9 & $\mathrm{~F}$ & 33 & Mitral stenosis and regurgitation & II & $580-1340$ & 925 & 208 & $4 \cdot 4$ & $1 \cdot 3$ \\
\hline I0 & $\mathbf{F}$ & 59 & Mitral stenosis & III & $600-1070$ & 830 & 212 & 3.9 & 0.8 \\
\hline II & $\mathrm{F}$ & 45 & Mitral stenosis and regurgitation & III & $400-840$ & - & 316 & - & $2 \cdot 0$ \\
\hline 12 & $\mathbf{F}$ & 65 & Ischaemic heart disease & II & $440-1130$ & 620 & 670 & 0.9 & $>10 \cdot 0$ \\
\hline 13 & $\mathbf{M}$ & 60 & Lone atrial fibrillation & I & $480-1140$ & 600 & 500 & $1 \cdot 2$ & $>10 \cdot 0$ \\
\hline 14 & $\mathrm{~F}$ & 34 & Lone atrial fibrillation & I & $420-840$ & 575 & 450 & $1 \cdot 3$ & $>10 \cdot 0$ \\
\hline 15 & $\mathbf{M}$ & 54 & Mitral regurgitation & IV & $420-900$ & 560 & 500 & $\mathbf{I} \cdot \mathbf{I}$ & $>10.0$ \\
\hline
\end{tabular}

NYHA $=$ New York Heart Association functional classification.

$R R$ range $=$ Shortest and longest $R R$ interval, msec.

' $T$ ' $=R R$ interval at maximum left ventricular ejection time, msec. See text.

' $S$ ' = Slope of rapid inflow phase of left ventricular ejection time RR plot, msec/sec.

$\mathrm{Rmv}=$ Mitral valve resistance index, $\mathrm{T} / \mathrm{S}$.

Diastolic closure rate $=$ Mitral echo diastolic closure rate, $\mathrm{cm} / \mathrm{sec}$.

\section{Left ventricular ejection time}

Left ventricular ejection time was measured from the initial rapid upstroke of the external carotid pulse tracing to the incisura.

\section{Pre-ejection period}

Pre-ejection period was obtained by subtracting the left ventricular ejection time from the $\mathrm{QA}_{2}$ interval, measured from the initial deflection of the QRS complex to the first high frequency vibrations of the aortic component of the second heart sound.

1 Pre-ejection period: left ventricular ejection time

The ratio of the pre-ejection period to left ventricular ejection time was calculated from the data derived above.

For each patient, the left ventricular ejection time was plotted against the preceding $R R$ interval measured in msec. Pre-ejection period and pre-ejection period/left ventricular ejection time ratios were plotted against the preceding RR interval in 9 of the cases studied. Between 25 and 50 values were obtained at varying cycle lengths for each graph.

\section{Comparison of patients}

Echograms of the anterior leaflet of the mitral valve were recorded in all patients (Smith-Kline Instrument Co. EKO-20), and diastolic closure rate was measured to the nearest $0.1 \mathrm{~cm} / \mathrm{sec}$ according to standard technique (Gustafson, 1967).

Values for maximum filling time ' $T$ ' and rapid inflow slope ' $S$ ' for each patient were measured from the left ventricular ejection time curve as defined in the next section and illustrated schematically in Fig. I. Values for ' $T$ ' and ' $S$ ' were plotted against increasing obstruction across the mitral valve as measured by decreasing diastolic closure rate of the mitral echogram. A mitral valve resistance index, $R m v$, in units, was defined by dividing the value for ' $T$ ' by the value for ' $S$ ' in each patient. These values for $\mathrm{Rmv}$ were compared with the mitral echogram.

\section{Results}

\section{Left ventricular ejection time}

In 14 of the patients studied, the graph of left ventricular ejection time against $R R$ interval formed a curve that was approximately linear over both the shorter and longer ranges of cycle length. These curves could easily be represented by two intersecting lines, as illustrated schematically in Fig. r.

At shorter RR intervals the left ventricular ejection time increased rapidly and linearly with increasing $R R$, as represented by line $A B$ in Fig. $I$. In each of these cases a value for $R R$ duration was reached beyond which no further increase in left ventricular ejection time occurred with longer cycle length, as represented by line BC. 


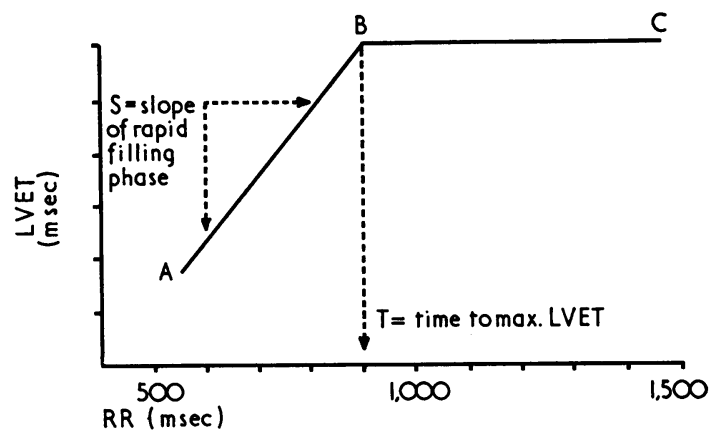

FIG. I Schematic representation of left ventricular ejection time vs. preceding $R R$ interval for individual patients. At shorter cycle lengths, the rapidly rising phase $A B$ defines a slope ' $S$ ' in $\mathrm{msec} / \mathrm{sec}$. $A$ value for filling time ' $T$ ' is defined as the RR interval in msec beyond which no significant increase in left ventricular ejection time occurs. Measurement of values for ' $T$ ' and ' $S$ ' allows comparison of patients independent of the absolute values of left ventricular ejection time.

A clearly biphasic graph could be shown in this way in 14 of the 15 cases independent of the variable absolute values for maximum left ventricular ejection time. In Fig. 2, this wide range is seen in the

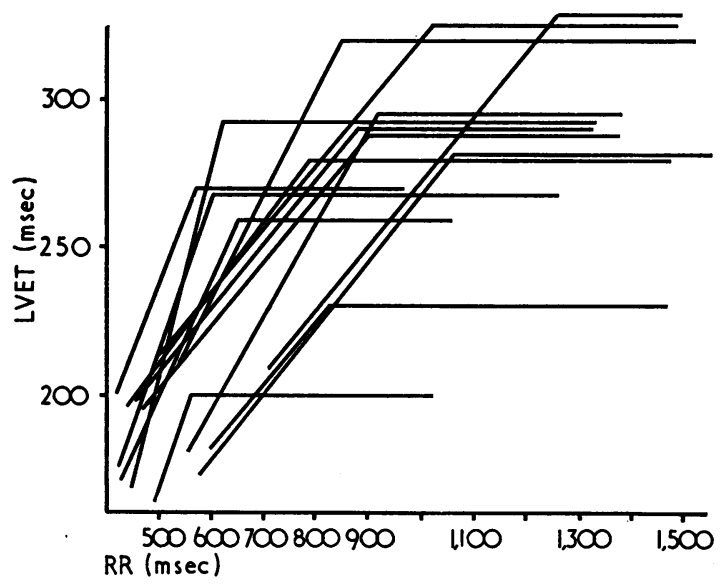

FIG. 2 Composite of schematic curves of left ventricular ejection time vs. preceding $R R$ interval. In each of these 14 cases a value for the $R R$ interval is reached beyond which no further significant increase in left ventricular time occurs. The range of absolute values for left ventricular ejection time is wide.

composite of these schematic curves of left ventricular ejection time plotted against the preceding $R R$ interval. In one of the patients with mitral stenosis (Case II), no RR interval exceeded 840 msec, and only a value for ' $S$ ' could be measured since no levelling was apparent in the absence of sufficiently long $R R$ intervals.

The filling time ' $T$ ' was defined as the $R R$ interval in msec at point B in Fig. I, being the intersection of the two linear phases of the left ventricular ejection curve. In addition, a slope ' $S$ ' in $\mathrm{msec} / \mathrm{sec}$ was defined as the incremental increase in left ventricular ejection time with $R R$ during the rapid inflow phase of diastole (AB in Fig. $\mathrm{I}$ ), occurring over the shorter range of $R R$ intervals.

Representative plots are illustrated in Fig. 3.

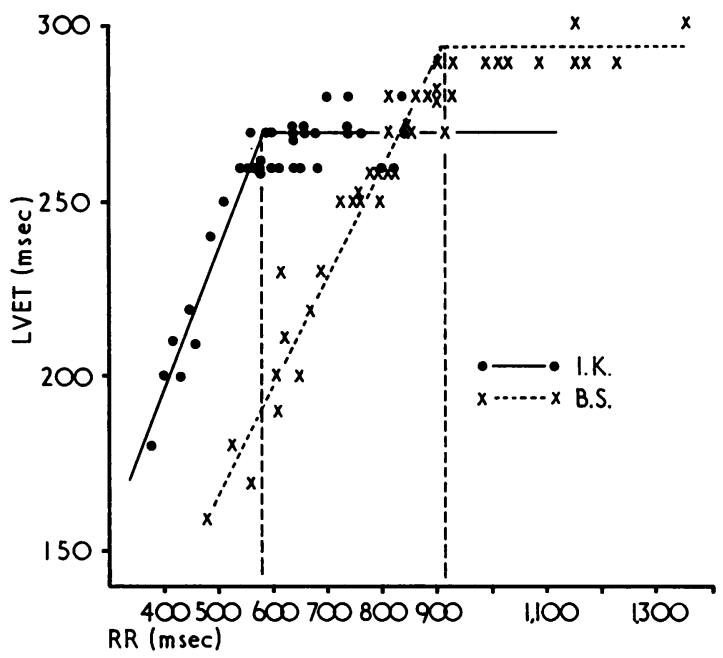

FIG. 3 Comparison of two patients. Case 14 (I.K.) with lone atrial fibrillation, the values for ' $T$ ' of 575 msec and for ' $S$ ' of $450 \mathrm{msec} / \mathrm{sec}$ are different from those of Case 4 (B.S.) with moderate mitral stenosis, for whom ' $T$ ' is longer at 915 msec and ' $S$ ' is reduced at $312 \mathrm{msec} / \mathrm{sec}$.

For Case 14, with lone atrial fibrillation and no mitral stenosis, maximum ejection time occurred at ' $T$ ' $=575 \mathrm{msec}$. Longer $R R$ intervals, and hence longer diastolic filling time, produced no further increase in left ventricular ejection time. During the phase of rapid ventricular inflow at short $R R$ intervals, the incremental increase in left ventricular ejection with time is measured as ' $S$ ' $=450 \mathrm{msec} /$ sec. For Case 4 by contrast, with moderate mitral stenosis, the value for ' $T$ ' is considerably longer at $915 \mathrm{msec}$, and the slope ' $S$ ' less at $312 \mathrm{msec} / \mathrm{sec}$.

Comparison of measured values for ' $T$ ' and ' $S$ ' for the patients studied (Fig. 4) shows a generally inverse relation. As filling time ' $T$ ' decreases, the slope ' $S$ ' of the rapid inflow phase increases. Patients without mitral stenosis, represented by closed circles, could be clearly distinguished from 


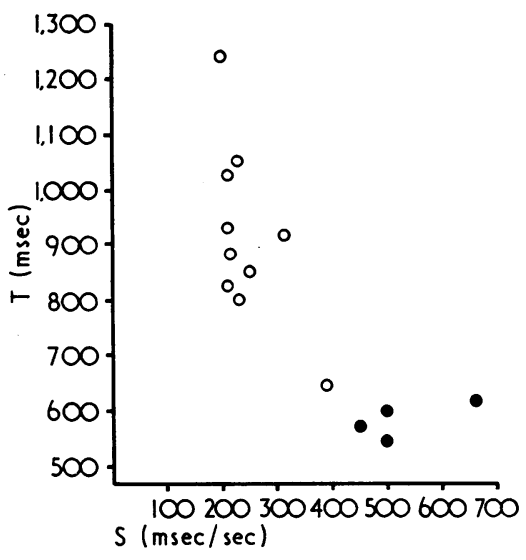

FIG. 4 A generally inverse relation exists between the value for ' $T$ ' and the value for ' $S$ ' for each patient. Patients with mitral stenosis (open circles) can be distinguished from those with no mitral stenosis (closed circles).

those with mitral stenosis both by high values for ' $S$ ' and low values for ' $T$ '.

\section{Comparison of patients}

All patients with mitral stenosis could be distinguished from those without mitral stenosis by

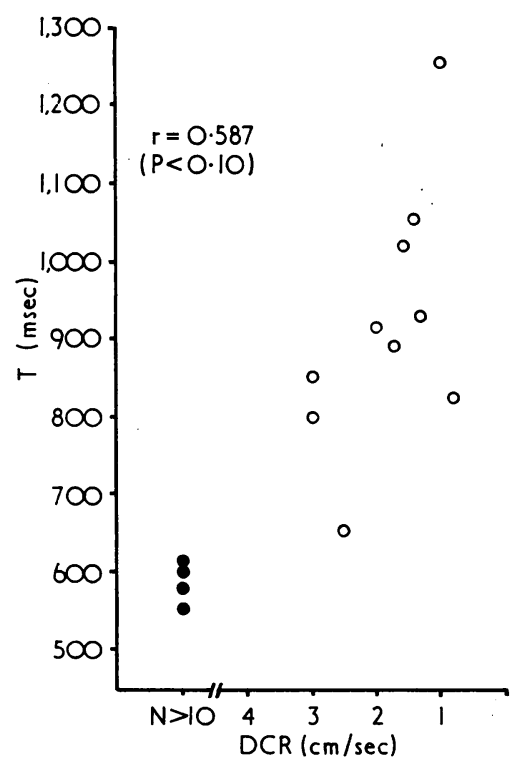

FIG. 5 Comparison of values for ' $T$ ' with the diastolic closure rate of the mitral echogram. In patients with mitral stenosis (open circles), ' $T$ ' increases with decreasing diastolic closure rate. $N=$ number of patients. values for ' $T$ ' in excess of $640 \mathrm{msec}$. In those patients with mitral stenosis, ' $T$ ' tended to increase with decreasing diastolic closure rate, though the relation is not clearly a linear one $(r=0.587, P<0.10)$, as seen in Fig. 5.

Patients with mitral stenosis all had values for ' $S$ ' that were less than $400 \mathrm{msec} / \mathrm{sec}$ and could be distinguished from those with no mitral stenosis, as seen in Fig. 6. In those with mitral stenosis, ' $S$ ' tended to decrease with decreasing diastolic closure rate in a generally linear manner $(r=$ $-0.664, P<0.05)$.

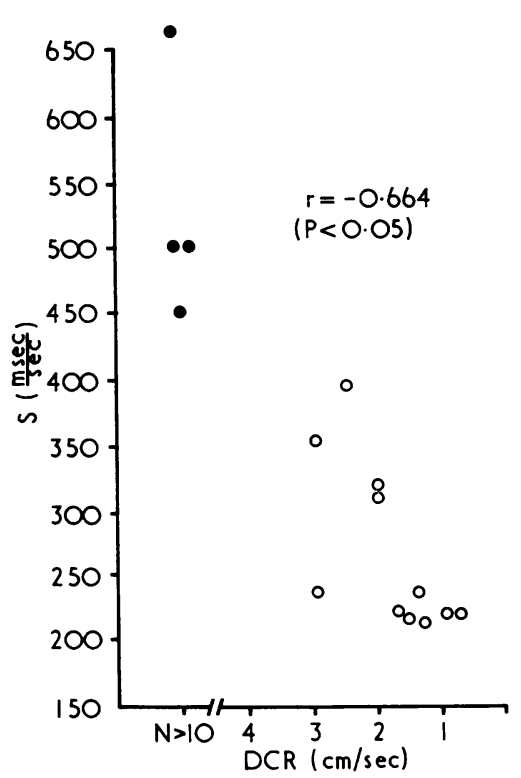

FIG. 6 Comparison of values for ' $S$ ' with the diastolic closure rate of the mitral echogram. Within the subgroup of patients with mitral stenosis (open circles), ' $S$ ' decreases with decreasing diastolic closure rate. $N=$ number of patients.

Values for Rmv, obtained by dividing ' $T$ ' by ' $S$ ' for each patient, are compared with diastolic closure rate in Fig. 7. In patients with no mitral valve stenosis, $\mathrm{Rmv}$ was less than $\mathrm{I} \cdot 4$ units. All patients with mitral stenosis had $R m v$ values ranging from $\mathrm{I} \cdot 6$ to 5.9 units which tended to increase in a linear manner with decreasing diastolic closure rate $(r=0.732, P<0.02)$.

\section{Pre-ejection period and pre-ejection period/ left ventricular ejection time}

These were compared with the preceding $R R$ interval in 9 patients. In Fig. 8, it can be seen that pre-ejection period decreased with increasing $R R$ over the range of shorter cycle lengths. Values for 


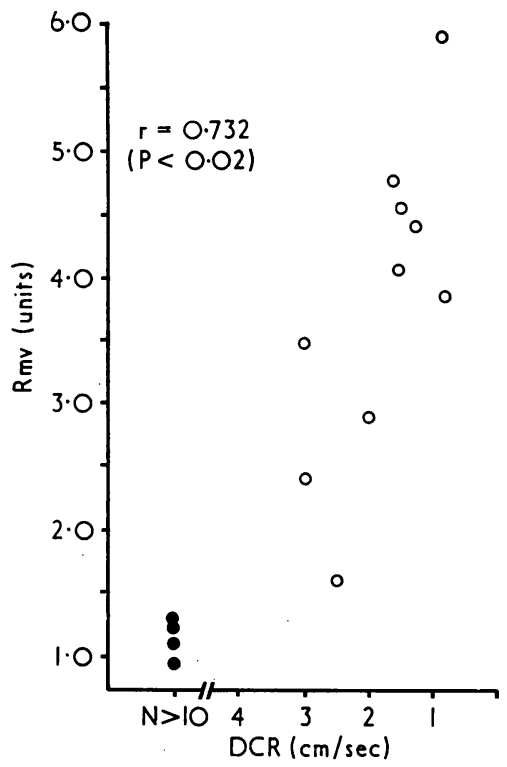

FIG. 7 Comparison of values for Rmv, derived by dividing the value for ' $T$ ' by the value for ' $S$ ' for each patient, with the diastolic closure rate of the mitral echogram. Patients with mitral stenosis (open circles) can be clearly distinguished from those without mitral stenosis (closed circles). In patients with mitral stenosis, Rmv increases in a generally linear manner with decreasing diastolic closure rate. $N=$ number of patients.

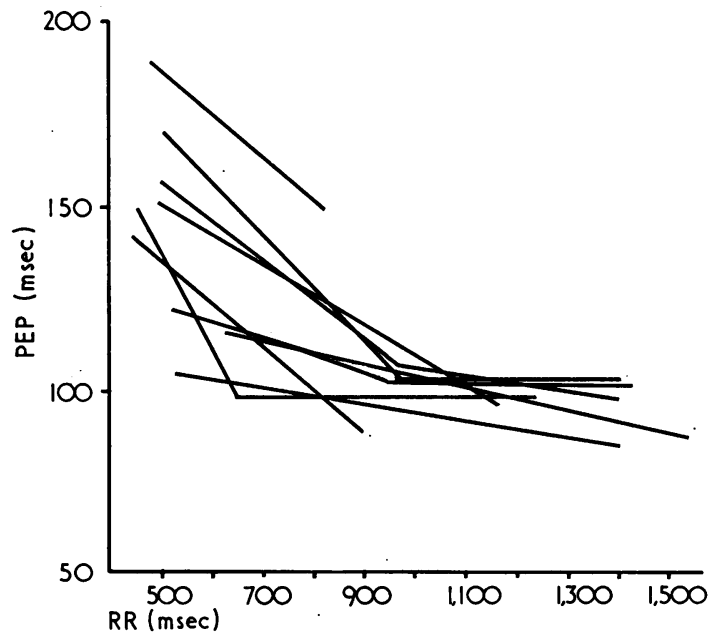

FIG. 8 Composite of curves of pre-ejection period vs. preceding $R R$ interval. Pre-ejection period decreases with increasing cycle length. pre-ejection period tended to reach a minimum at RR intervals corresponding with the filling time ' $T$ ' for the patient in 4 of the cases, but in the others only a continuously decreasing trend could be demonstrated over the entire RR range. Since variation in pre-ejection period values tended to be small, it is likely that this discrepancy is due to limitation of accuracy in measurement.

The ratio of pre-ejection period to left ventricular ejection time decreased rapidly and linearly with increasing cycle length over the range of shorter cycles to reach a minimum value at the $R R$ interval closely corresponding to the value for ' $\mathrm{T}$ ' for the patient. This is consistent with the predominant effect of left ventricular ejection time change in determining the value of the ratio of pre-ejection period to left ventricular ejection time.

\section{Discussion}

In 1906, Henderson, Scarbrough, and Chillingworth observed that 'since the ventricles can discharge at each systole only so much blood as enters their chambers during diastole, the principles controlling and the conditions determining the diastolic filling of the ventricles control and determine the variations in the volume of the blood which is discharged into the arteries at each heart beat.'

Extending this work, Wiggers (I92I) showed a rapid inflow phase of early diastole, where rapid filling of the ventricles takes place until equalization of pressure occurs or a subsequent contraction interrupts the filling. Both these workers noted that there was little, if any, further increase in ventricular filling during the subsequent period of diastasis which preceded atrial contraction. Lombard and Cope (I926), comparing the relative durations of systole, diastole, and preceding cycle length in groups of normal men in sinus rhythm, showed that about $450 \mathrm{msec}$ between cycles is required to allow completion of the period of rapid ventricular inflow.

From this early work, it would be expected that in patients with atrial fibrillation, obstruction to left ventricular inflow should prolong the time required to achieve complete ventricular filling. Furthermore, over the range of shorter $R R$ intervals where subsequent systoles interrupt the early rapid inflow phase of diastole at varying points, the change in ventricular filling with time should reflect the flow across the obstructed or non-obstructed mitral valve.

The left ventricular ejection time is an easily obtainable parameter of stroke volume which in a single patient under constant conditions should reflect changes in ventricular filling volume. Most 
work on this subject has been concerned with average values for groups of subjects rather than variation within individuals. Wiggers was aware of the lengthening of ejection caused by increasing venous return, and Lombard and Cope showed a linear increase in the duration of systole with increasing cycle length in groups of normal subjects. More recent work (Weissler, Peeler, and Roehll, 196I) has shown close correlation between left ventricular ejection time and measured stroke volume for groups of normal subjects which was also applicable to patients with mitral valve disease, though patients with aortic regurgitation were found to have higher absolute values for left ventricular ejection time relative to stroke volume. In individual patients with atrial fibrillation, Greenfield et al. (1968) found a close correlation between duration of ejection and stroke volume measured by pressure-gradient technique, though the relation was not strictly linear.

Katz and Feil (1923) plotted ejection time against $R R$ interval for a heterogeneous group of patients in atrial fibrillation and showed that ejection times increased with increasing cycle length, and also tended to be shorter than in subjects in sinus rhythm at similar heart rates. Greenfield and coworkers noted a curvilinear increase in both stroke volume and duration of ejection with increasing $R R$ interval in another heterogeneous group with atrial fibrillation, but no observations in individual cases were made. That individual patients with atrial fibrillation without mitral stenosis tend to achieve a maximal left ventricular ejection time was noted by Tavel et al. (1972), who plotted left ventricular ejection time against heart rate rather than RR interval. The different value for the cycle length at which left ventricular ejection time levelling occurred in the absence of mitral stenosis (850 msec vs. $600 \mathrm{msec}$ in the present study) may be due to the flattening effect on the left ventricular ejection time slope resulting from using heart rate on the time axis. The shortening of the time axis at longer RR interval ranges when heart rate is used may also account for the absence of left ventricular ejection time levelling at long cycle lengths for patients with mitral stenosis in their work, since quite long RR intervals would be required before this effect would be noted.

The results of the present study confirm the expectation that in patients with mitral stenosis and atrial fibrillation, a value for $R R$ interval exists beyond which no further significant increase in left ventricular ejection occurs. With the exception of the very shortest cycle lengths, the total duration of systole as measured by the $\mathrm{QA}_{2}$ interval remained within narrow limits, a finding also noted by Green- field et al., so that an increasing RR interval effectively represents increasing diastole.

This study differs from previous work in focusing on changes in individual patients, thereby using each patient as his own relative control. It assigns greater importance to the extremes of the RR interval range for each patient, which enable the left ventricular ejection curve to be schematically represented as two intersecting lines from which further information may be derived independent of the variable absolute value for maximum left ventricular ejection time.

It is shown that an index of mitral valve resistance, Rmv, can be derived from the external carotid pulse tracing and electrocardiogram of patients in atrial fibrillation. This index clearly distinguishes patients with mitral stenosis from those with no obstruction to left ventricular inflow and correlates reasonably well with decreasing mitral diastolic closure rate as measured by echocardiography. Values for ' $T$ ' and ' $S$ ' could also be used to distinguish between patients with and without mitral stenosis but were not as well correlated with the mitral echogram.

The diastolic closure rate of the mitral echogram correlates well with mitral valve orifice size (Edler, 1967; Gustafson, 1967) and has been used for comparisons within the group of patients with mitral stenosis. Since only 3 of the patients in this series underwent cardiac catheterization and haemodynamic data are not available for the group, the mechanism by which $R m v$ reflects mitral valve obstruction is largely speculative in this report.

Wood noted in I954 that a useful index of mitral stenosis could be obtained from the mean left atrial pressure and the cardiac output measured at catheterization. Parameters of these functions obtained in a non-invasive manner would be expected to produce a similarly useful index. Though purely speculative at the present time, it might be suggested that the time to maximum ventricular filling ' $T$ ' represents the time required for equilibration of left atrial and left ventricular pressures. This should be in part dependent on the pressure gradient across the mitral valve, as well as atrial volume and valve area. Values for ' $S$ ', the change in left ventricular ejection time with time during the shorter RR intervals, should represent some parameter of flow across the mitral valve. Rmv therefore includes indirectly measured postulated parameters of both flow and pressure gradient across the valve.

There are numerous theoretically valid objections to this oversimplified construction. The Poiseuille relation on which it is based is not strictly applicable to flow across a stenotic mitral valve, since kinetic energy loss is high across thin projections, the rate of flow is not steady, the flow is not 
laminar, and the orifice size and configuration are not constant (McDonald, 1960; Gorlin and Gorlin, I95I). Obstruction to inflow resulting from poor compliance is not considered. Despite these objections, the correlation of $\mathrm{Rmv}$ and the mitral echogram suggests that evaluation of the left ventricular ejection curve may be a useful technique for the noninvasive assessment of mitral stenosis in the presence of atrial fibrillation. Haemodynamic correlations will be of interest, particularly if information regarding pulmonary vascular resistance and pressure gradient across the valve can be extracted from measured values of ' $T$ ' and ' $S$ '.

The pre-ejection period comprises both the interval from the onset of the QRS complex to mitral closure $\left(\mathrm{QS}_{1}\right)$ and the isometric contraction period preceding systolic ejection. No attempt was made in this study further to define characteristics of the pre-ejection period and pre-ejection period/left ventricular ejection time curves, both of which were found to decrease with increasing $R R$ interval. A decrease in pre-ejection period with increasing cycle length can also be seen in the work of Greenfield et al. (1968). Wiggers in I92 I showed that increased venous return shortens the isometric contraction period in sinus rhythm, and Katz and Feil in 1923 found a similar response with increasing $R R$ interval for groups of patients in atrial fibrillation. Messer et al. (I95I) demonstrated that in mitral stenosis the $Q S_{1}$ interval shortens as cycle length increases and the pressure gradient across the valve decreases, a finding also noted by Wells (1954).

Sherman and Lewis (1972) have recently observed that the pre-ejection period/left ventricular ejection time ratios decrease with increasing cycle length, a result confirmed in this study. The variation of pre-ejection period with cycle length for individual patients is consistent with the shortening of isometric contraction time which accompanies increasing preload but is opposite in direction from that which would be predicted from equivalent heart rates in sinus rhythm from the group data of Weissler et al. (1969). This difference might be explained by higher sympathetic tone accompanying faster heart rate in data derived from groups of subjects in sinus rhythm.

Although variations in pre-ejection period and pre-ejection period time left ventricular ejection ratio have been related to changes in various indices of contractility (Garrard, Weissler, and Dodge, 1970; Martin et al., 1971; Ahmed et al., 1972), this does not appear to be valid in the presence of alterations in preload (Shah et al., 1969; Braunwald, 1971). From the nearly reciprocal relation of left ventricular ejection time and pre-ejection period changes with cycle length, it seems likely that the pre-ejection period and pre-ejection period/left ventricular ejection time changes with increasing $R R$ interval reflect both the improving ventricular performance resulting from increasing filling and the diminishing pressure gradient across the mitral valve.

Appreciation is extended to Dr. Aubrey Leatham for review and criticism of the manuscript.

\section{References}

Ahmed, S. S., Levinson, G. E., Schwartz, C. J., and Ettinger, P. O. (1972). Systolic time intervals as measure of the contractile state of the left ventricular myocardium in man. Circulation, 46, 559.

Braunwald, E. (I97I). On the difference between the heart's output and its contractile state. Circulation, 43, 171 .

Edler, I. (1967). Ultrasoundcardiography in mitral valve stenosis. American fournal of Cardiology, 19, 18.

Garrard, C. L., Jr., Weissler, A. M., and Dodge, H. T. (1970). The relationship of alterations in systolic time intervals to ejection fraction in patients with cardiac disease. Circulation, 42, 455.

Gorlin, R., and Gorlin, S. G. (195I). Hydraulic formula for calculation of the area of the stenotic mitral valve, other cardiac valves, and central circulatory shunts. American Heart Fournal, 4I, $\mathrm{I}$.

Greenfield, J. C., Jr., Harley, A., Thompson, H. K., and Wallace, A. G. (1968). Pressure-flow studies in man during atrial fibrillation. Fournal of Clinical Investigation, 47, 24 II.

Gustafson, A. (1967). Correlation between ultrasoundcardiography, haemodynamics, and surgical findings in mitral stenosis. American fournal of Cardiology, 19, 32.

Henderson, Y., Scarbrough, M. McR., and Chillingworth, F. P. (1906). The volume curve of the ventricles of the mammalian heart, and the significance of this curve in respect to the mechanics of the heart-beat and the filling of the ventricles. American fournal of Physiology, 16, 325.

Katz, L. N., and Feil, H. S. (1923). Clinical observations on the dynamics of ventricular systole. (I) Auricular fibrillation. Archives of Internal Medicine, 32, 672.

Lombard, W. P., and Cope, O. M. (I926). The duration of the systole of the left ventricle of man. American fournal of Physiology, 77, 263.

McDonald, D. A. (1960). Blood Flow in Arteries. Edward Arnold, London.

Martin, C. E., Shaver, J. A., Thompson, M. E., Reddy, P. S., and Leonard, J. J. (1971). Direct correlation of external systolic time intervals with internal indices of left ventricular function in man. Circulation, 44, 419.

Messer, A. L., Counihan, T. B., Rappaport, M. B., and Sprague, H. B. (I95I). The effect of cycle length on the time of occurrence of the first heart sound and the opening snap in mitral stenosis. Circulation, 4, 576.

Schoenfeld, C. D., Cohen, S., Taguchi, J. T., and Weissler, A. M. (1963). Relationships between cycle length and left ventricular ejection in atrial fibrillation. Clinical Research Proceedings, II, 26.

Shah, P. M., Tager, I. B., Shaefer, R. A., and Kramer, D. H. (1969). Determinants of pre-ejection period of left ventricle in the assessment of myocardial performance in man. Clinical Research, 17, 263.

Sherman, J. A., and Lewis, R. P. (1972). Systolic time intervals in atrial fibrillation. Circulation, 46, Suppl. II. II-220. 
Tavel, M. E., Baugh, D. O., Feigenbaum, H., and Nasser, W. K. (1972). Left ventricular ejection time in atrial fibrillation. Circulation, 46, 744.

Weissler, A. M., Harris, W. S., and Schoenfeld, C. D. (1969). Bedside techniques for the evaluation of ventricular function in man. American fournal of Cardiology, 23, 577.

Weissler, A. M., Peeler, R. G., and Roehll, W. H., Jr. (I96r). Relationships between left ventricular ejection time, stroke volume, and heart rate in normal individuals and patients with cardiovascular disease. American Heart fournal, 62, 367.

Wells, B. (1954). The assessment of mitral stenosis by phonocardiography. British Heart fournal, 16, 261.
Wiggers, C. J. (1921). Studies on the consecutive phases of the cardiac cycle. (I) the duration of the consecutive phases of the cardiac cycle and the criteria for their precise determination. (II) The laws governing the relative durations of ventricular systole and diastole. American fournal of Physiology, 56, 415 and 439.

Wood, P. (1954). An appreciation of mitral stenosis. British Medical fournal, I, IOSI and III3.

Requests for reprints to Dr. Paul Kligfield, Division of Cardiology, Department of Medicine, New York Hospital - Cornell Medical Center, 525 East 68th Street, New York, N.Y. roo2r, U.S.A. 